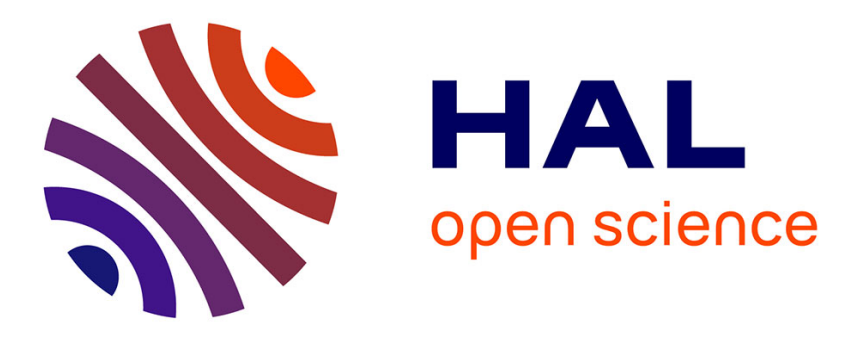

\title{
High-resolution electrophoretic separation and integrated-waveguide excitation of fluorescent DNA molecules in a lab on a chip
}

Chaitanya Dongre, Jasper van Weerd, Rob van Weeghel, Rebeca Martinez Vazquez, Roberto Osellame, Giulio Cerullo, Marina Cretich, Marcella Chiari, Hugo J.W.M. Hoekstra, Markus Pollnau

\section{To cite this version:}

Chaitanya Dongre, Jasper van Weerd, Rob van Weeghel, Rebeca Martinez Vazquez, Roberto Osellame, et al.. High-resolution electrophoretic separation and integrated-waveguide excitation of fluorescent DNA molecules in a lab on a chip. Electrophoresis, 2010, 31 (15), pp.2584. 10.1002/elps.201000126 . hal-00599463

\section{HAL Id: hal-00599463 https://hal.science/hal-00599463}

Submitted on 10 Jun 2011

HAL is a multi-disciplinary open access archive for the deposit and dissemination of scientific research documents, whether they are published or not. The documents may come from teaching and research institutions in France or abroad, or from public or private research centers.
L'archive ouverte pluridisciplinaire HAL, est destinée au dépôt et à la diffusion de documents scientifiques de niveau recherche, publiés ou non, émanant des établissements d'enseignement et de recherche français ou étrangers, des laboratoires publics ou privés. 
High-resolution electrophoretic separation and integratedwaveguide excitation of fluorescent DNA molecules in a lab on a chip

\begin{tabular}{|r|l|}
\hline Journal: & Electrophoresis \\
\hline Manuscript ID: & elps.201000126.R1 \\
\hline Date - Manuscript type: & Research Paper \\
\hline Author: & 30 -Apr-2010 \\
& $\begin{array}{l}\text { Complete List of Authors: } \\
\text { Nartinez Vazquez, Rebeca; Politecnico di Milano } \\
\text { Nan Weerd, Jasper; Zebra Bioscience BV } \\
\text { Osellame, Roberto; Politecnico di Milano } \\
\text { Cerullo, Giulio; Politecnico di Milano } \\
\text { Cretich, Marina; ICRM-CNR } \\
\text { Chiari, Marcella; ICRM-CNR } \\
\text { Hoekstra, Hugo; University of Twente } \\
\text { Pollnau, Markus; University of Twente }\end{array}$ \\
\hline Keywords: & integrated optofluidics, lab on a chip, fluorescence, ultrasensitive \\
\hline
\end{tabular}

\section{s scholaroNE" \\ Manuscript Central}




\section{High-resolution electrophoretic separation and}

\section{integrated-waveguide excitation of fluorescent DNA}

\section{molecules in a lab on a chip}

Chaitanya Dongre, ${ }^{1, *}$ Jasper van Weerd, ${ }^{2}$ Rob van Weeghel, ${ }^{2}$ Rebeca Martinez Vazquez, ${ }^{3}$ Roberto Osellame, ${ }^{3}$ Giulio Cerullo, ${ }^{3}$ Marina Cretich, ${ }^{4}$ Marcella Chiari, ${ }^{4}$

Hugo J.W.M. Hoekstra, ${ }^{1}$ and Markus Pollnau ${ }^{1}$

${ }^{1}$ Integrated Optical Microsystems Group, MESA+ Institute for Nanotechnology, University of Twente, P.O. Box 217, 7500 AE Enschede, The Netherlands

*Corresponding Author Email: C.Dongre@ewi.utwente.nl, Fax: +31 534893343

${ }^{2}$ Zebra Bioscience BV, Wethouder Beversstraat 185, 7543 BK Enschede, The Netherlands

${ }^{3}$ Istituto di Fotonica e Nanotecnologie (IFN)-CNR, Dipartimento di Fisica-Politecnico di Milano,

Piazza Leonardo da Vinci 32, 20133 Milan, Italy

${ }^{4}$ Istituto di Chimica del Riconoscimento Molecolare (ICRM) - CNR,

Via Mario Bianco 9, 20131 Milan, Italy

List of abbreviations: Laser induced fluorescence (LIF), Microfluidic (MF), lab on a chip (LOC), Capillary electrophoresis (CE), Waveguide (WG), Limit of detection (LOD), epoxy-poly-(dimethylacrylamide) (EPDMA), Hydroxyethyl cellulose (HEC), Photomultiplier tube (PMT)

Keywords: fluorescence, integrated optofluidics, lab-on-a-chip, ultrasensitive 


\begin{abstract}
By applying integrated waveguide laser excitation to an optofluidic chip, fluorescently labeled DNA molecules of 12 or 17 different sizes are separated by capillary electrophoresis with high operating speed and low sample consumption of $\sim 600$ picoliters. When detecting the fluorescence signals of migrating DNA molecules with a photomultiplier tube, the limit of detection is as low as 2.1 picomolar. In the diagnostically relevant size range $(\sim 150-1000$ base-pairs $)$ the molecules are separated with reproducibly high sizing accuracy (> 99\%) and the plug broadening follows Poissonian statistics. Variation of the power dependence of migration time on base-pair size - probably with temperature and condition of the sieving gel matrix - indicates that the capillary migration cannot be described by a simple physical law. Integrated waveguide excitation of a $12-\mu \mathrm{m}$ narrow microfluidic segment provides a spatiotemporal resolution that would, in principle, allow for a 20 -fold better accuracy than is currently supported by state-of-the-art electrophoretic separation in microchips, thereby demonstrating the potential of this integrated optical approach to fulfill the resolution demands of future electrophoretic microchips.
\end{abstract}




\section{Introduction}

Microfluidic (MF) lab-on-a-chip (LOC) devices [1,2] have enabled integrated DNA sequencing [3] and genetic diagnostics [4, 5]. Capillary electrophoresis (CE) separation is a powerful method over a broad range of molecular sizes, thanks to flexible MF protocols, exploiting a variety of sieving gel matrices. The inherent advantages of MF $\mathrm{CE}$, high-speed operation and low reagent volumes, can be combined with laser-induced fluorescence (LIF) detection [6-8], resulting in optofluidic integration [9, 10] toward compact, sensitive on-chip bio-analysis tools [11] which aim at solving real-life challenges in medicine [12], e.g. identification of genomic deletions or insertions associated with genetic illnesses.

Fluorescence excitation via an integrated optical waveguide (WG) provides advantages over focusing an external laser beam by lenses or through a microscope, such as stability and reproducibility of the optical excitation and avoidance of the requirement to realign the laser beam from time to time. Besides, it is one step toward full sensor integration in the MF LOC. We apply the technique of femtosecond-laser micromachining $[13,14]$ to post-process optical WGs into commercially mass-produced LOCs on a fast, flexible, chip-by-chip basis [15], thereby exploiting the existing mature MF fabrication infrastructure, while achieving integrated fluorescence excitation. Having pioneered the use of such integrated-WG laser excitation for electrophoretic DNA analysis [16], here we demonstrate excellent DNA-sizing accuracy and low background noise, resulting in a lower limit of detection (LOD) compared to earlier accounts [17]. Furthermore, the spatio-temporal resolution provided by integrated waveguide excitation 
can allow for a 20-fold improvement in accuracy if better MF CE separation protocols become available.

\section{Optofluidic chip fabrication and characterization}

The lay-out of our optofluidic chip is presented in Fig. 1. Chips were fabricated in a twostep procedure. Firstly, the MF channel network and MF reservoirs were patterned photolithographically and wet-etched in fused silica glass and then sealed off by bonding another piece of fused silica glass on top. The chip has dimensions of $55 \mathrm{~mm} \times 5.5 \mathrm{~mm} \times$ $1 \mathrm{~mm}$ and the MF channels have a cross section of $\sim 110 \mu \mathrm{m}$ width and $\sim 50 \mu \mathrm{m}$ depth (LioniX BV) [http://www.lionixbv.nl].

In a second step, the optical WG was inscribed into the bulk of such a fused silica chip by femtosecond-laser writing at a translation speed of $20 \mu \mathrm{m} / \mathrm{s}$, using a Ti:Sapphire laser operating at $800 \mathrm{~nm}$ wavelength with $150 \mathrm{fs}, 4 \mu \mathrm{J}$ pulses at a repetition rate of 1 kHz. Employing astigmatic beam shaping, an elliptical cross section of the written WG was obtained, with a major diameter of $\sim 50 \mu \mathrm{m}$ in the vertical direction, in order to excite the maximum possible volume of the MF channel, while the minor diameter in the horizontal direction is $\sim 12 \mu \mathrm{m}$ in order to retain a high spatial resolution along the direction of DNA flow and separation. The WG displays a graded refractive index profile and a maximum refractive index increase of $2 \times 10^{-3}$. Fiber-to-chip coupling of laser light with coupling losses of $<2 \mathrm{~dB}$ was achieved and the WG propagation losses were measured to be in the range of $0.5-0.9 \mathrm{~dB} / \mathrm{cm}$ at the wavelength of $543 \mathrm{~nm}$. 
The excitation WG intersects the CE separation channel orthogonally at a distance of $\sim 3.6 \mathrm{~cm}$ from the MF crossing junction at which the separation commences, toward the end of the MF CE separation channel, close to reservoir 4, where the plug separation is highest. The fluorescence signal was detected through a small detection window in order to reduce the background noise. Constant, inherent, mutual alignment of the excitation WG with its detection window has the potential to render our system more compact, faster to operate, and more reproducible compared to conventional LIF approaches [6-8].

\section{Results and discussion}

\subsection{Electrophoretic separation protocol}

Prior to the experimental runs the inner walls of the MF channel network were coated with an epoxy-poly-(dimethylacrylamide) (EPDMA)-based polymer in order to suppress the electro-osmotic flow and to minimize adsorption of DNA molecules on the MF channel wall. Subsequently, the channels were filled with a sieving gel matrix consisting of hydroxyethyl-cellulose (HEC) (2\% wt./vol.), dissolved in $20 \mathrm{mM}$ MES / $20 \mathrm{mM}$ His buffer ( $\mathrm{pH}$ 6.2) in order to maximize the resolution of the DNA CE separation. SYBR Green I (Molecular Probes Inc.) was added according to the manufacturer's protocol. The reagents were sterilized, filtered $(0.22 \mu \mathrm{m}$ pore size $)$ and stored at $269 \mathrm{~K}$. The CE sample loading and separation protocol was based on actuation voltages of up to $1.5 \mathrm{kV}$, delivered by Pt electrodes integrated into the MF reservoirs, with the help of a Labview 
(National Instruments, Inc.) program to steer the MF control system (CapiliX BV) [http://www.capilix.com]. Application of a high voltage forced the negatively charged DNA molecules to migrate into the $\mathrm{CE}$ injection channel from sample reservoir 1 to waste reservoir 2. By switching the voltages at all four reservoirs simultaneously a wellconfined plug of DNA molecules - with a volume of $~ 605$ picoliters at the crossing junction of the two MF channels - was injected into the CE separation channel, from the MF crossing junction toward waste reservoir 4, and the DNA molecules contained in the plug volume were separated according to their size.

\subsection{Fluorescence excitation and detection}

Double-stranded DNA molecules were injected as a single mixture plug into the MF CE separation channel. For the movie of Fig. $2 \mathrm{Hg}$ arc lamp illumination was applied in order to visualize migration of the plugs, and the fluorescence was recorded by a CCD camera. Excitation through the optical WG was provided by the 488-nm line from a continuouswave Ar-ion laser. For ultrasensitive fluorescence detection, a photon-counting photomultiplier tube (PMT) (H7421-40, Hamamatsu Photonics K.K.) cooled down to 203 $\mathrm{K}$ was built onto the output port of an inverted microscope (DM5000, Leica Microsystems $\mathrm{GmbH}$ ) and aligned to collect the fluorescence signal through the detection window. Combination of excitation/emission band filters (K3, Leica Microsystems $\mathrm{GmbH}$, and XF57, Omega Optical, Inc.) in the fluorescence collection path ensured that only the fluorescence signal emitted by the fluorescently labeled DNA molecules reached 
the PMT, while all other sources of background signal, including the scattered laser excitation signals, were rejected.

A commercial DNA ladder consisting of molecules with 17 different sizes (in the range of 50-3000 bp) was injected, separated, and the individual plugs detected. The movie (Fig. 2, online) depicts the flow of the separated DNA plugs across the excitation WG under background $\mathrm{Hg}$ arc lamp illumination. The four snapshots in Fig. 2 correspond to the flow of the first well-separated DNA plug across the excitation WG. At this point in time, the remaining well-separated 16 plugs were not yet in the field of view of the CCD camera. Upon laser excitation of such a plug through the WG a sharp fluorescent segment only $\sim 12 \mu \mathrm{m}$ in width (matching well with the minor diameter of the WG crosssection) can be observed along the WG-MF-channel intersection (Fig. 2). Separation of these 17 DNA molecules was repeated in the same microchip, providing reproducible results, as shown in Fig. 3A-B.

After fitting the fluorescence intensity peaks in Fig. 3A-B with Gaussian profiles (the Gaussian fit of the peaks in Fig. 3B is shown in Fig. 3C) the obtained peak widths were plotted vs. the square root of the DNA molecule sizes, see Fig. 3D. The square-root dependence, confirmed by the linear fit, indicates that the migration of DNA molecules follows Poissonian statistics, the dispersion being governed by the number of collisions a molecule typically undergoes as it traverses through the sieving gel matrix [18]. The Gaussian fit is nevertheless justified, as for high expectation values the Poissonian approaches a Gaussian shape.

The LOD of DNA analysis in the integrated optofluidic chip were explored under integrated-WG laser excitation. Replacing the CCD camera by an ultrasensitive PMT 
allowed us to record the fluorescence intensity vs. migration time, as depicted in the electropherograms of Fig. 3A-B. We estimate the LOD by considering that an injected DNA concentration of $10 \mathrm{nM}$ diluted by, firstly, the spatial separation of 17 different molecule sizes and, secondly, plug broadening from $110 \mu \mathrm{m}$ to at least $248 \mu \mathrm{m}$, provided a fluorescence signal which is 125 times the four-fold standard deviation of the detected background noise. Based on this method [19], our conservative estimate of the LOD is 2.1 pM, which surpasses previously reported LOD values in laboratory setups involving on-chip-integrated fluorescence monitoring $[15,17]$.

\subsection{Base-pair resolution}

In an independent experiment carried out in a different microchip, another set of doublestranded DNA molecules with 12 different sizes, in a relatively smaller size range (150$500 \mathrm{bp}$ ), was separated and the individual plugs detected. The resulting electropherogram is shown in Fig. 4.

Optimization of the coating and sieving gel matrix led to excellent CE separation of the DNA molecules under integrated-WG laser excitation in both experiments, see Figs. 3A-B and 4. When plotting the temporal occurrence of the fluorescence peaks of the 17 molecules in Fig. 3A-B vs. their a priori known base-pair sizes, the two smallest (insufficient interaction with the sieving matrix) as well as the two largest DNA molecules (tendency to fold) exhibit clear deviations, while the peaks of all DNA molecules with sizes of 150-1000 base-pairs, in the diagnostically relevant region, are resolved with a sizing accuracy higher than 99\% (Fig. 5A). This high sizing accuracy is 
verified when analysing the result of Fig. 4 for the 12 DNA molecules in a smaller size range. The high sizing accuracy (>99\%) is comparable to state-of-the-art performance in microchip CE-based DNA sequencing without integrated-WG laser excitation [20, 21].

Each measured fluorescence peak in the electropherogram is a convolution along the flow axis - of the Gaussian WG mode-field cross-section $\Delta x_{W G}$ and the diffusion-induced physical width $\Delta x_{M F}$ of the MF plug. The resulting spatial plug widths $\Delta x$ are related to the temporal electropherogram peak widths $\Delta t$ (provided in Fig. 3D as full width at half maximum) as

$$
\Delta x=\frac{L}{t} \Delta t=\sqrt{\Delta x_{W G}^{2}+\Delta x_{M F}^{2}}
$$

where $L=3.6 \mathrm{~cm}$ and $t$ (data on the abscissa of Fig. 3A-C) are the migration length and time, respectively. In our experiment, the minimum electropherogram peak width was found to be $\Delta x=248 \mu \mathrm{m}$ (the very first peak in Fig. 3B-C). With $\Delta x_{W G}=12 \mu \mathrm{m}$, we obtain $\Delta x_{M F}=247.7 \mu \mathrm{m}>>\Delta x_{W G}$, i.e., the current spatial resolution is limited by the MF plug width, while the obtained integrated-WG mode profile would allow for a 20 -fold better resolution. MF technological advances, e.g. by improving the MF channel-wall coatings and sieving gel matrices, will lead to smaller MF plug widths, thereby fully exploiting the monitoring resolution achieved with integrated optical WGs. Clearly, integrated optical instrumentation is well suited to handle the resolution demands of future MF-chip generations. 
From the measured migration time $t$ vs. DNA size (Fig. 5A) we derive the electrophoretic mobility $\mu$ according to the equation

$$
\mu=\frac{L}{E t}
$$

where $L$ is the distance between the MF crossing junction and the detection window and $E$ is the electric field strength in the MF CE separation channel. The observed linear decrease (on double logarithmic scale) of the electrophoretic mobility with increasing DNA size (Fig. 5B) matches well with observations in conventional bulk capillary-based DNA gel electrophoresis setups [22].

By plotting the results of Fig. 5A on a double-logarithmic scale, see Fig. 5C, slopes of 0.24 and 0.25 (indicated by the straight lines) are derived for the two consecutive experiments, respectively, investigating the flow of the 17 DNA molecules. These results are significantly different from the slope of 0.31 derived for the flow of the 12 DNA molecules, despite the fact that in all cases a high sizing accuracy was obtained. This variation of power dependence points toward different flow mechanisms in these experiments, potentially depending on ambient temperature and/or condition of the sieving gel matrix, a result that questions whether a general, simple model of electrophoretic flow in micro-capillaries can be established and universally applied.

\section{Concluding remarks}

Simple and straight-forward integration of optical waveguides into commercial microfluidic chips by femtosecond-laser post-processing enables separation of fluorescently labeled DNA molecules with low limit of detection of $2.1 \mathrm{pM}$ and high 


\begin{abstract}
sizing accuracy of $>99 \%$, leaving plenty of room for further improvement of accuracy via better electrophoretic separation protocols. This fabrication technique also allows for three-dimensional waveguide structures [23], thereby further enhancing the potential of the optofluidic chip technology for monitoring on-chip CE.
\end{abstract}


The authors acknowledge fruitful discussions with R. Dekker, G. A. J. Besselink, and R. G. Heideman, technical support from A. J. F. Hollink, H. A. G. M. van Wolferen, N. Bellini, and M. J. Gilde, and provision of LOC platform by H. H. van den Vlekkert. Financial support was provided by the European Commission under FP6 project contract IST-2005-034562 - Hybrid Integrated Biophotonic Sensors Created by Ultrafast Laser Systems (HIBISCUS). 


\section{References}

[1] Whitesides, G. M., "The origins and the future of microfluidics," Nature 2006, 442, 368-373.

[2] Manz, A., Graber, N., Widmer, H. M., "Miniaturized total chemical analysis systems: A novel concept for chemical sensing," Sens. Actuators B 1990, 1, 244248.

[3] Eid, J., et al., "Real-time DNA sequencing from single polymerase molecules," Science 2009, 323, 133-138.

[4] Lagally, E. T., Mathies, R. A., "Integrated genetic analysis microsystems," J. Phys. D: Appl. Phys. 2004, 37, 245-261.

[5] Easley, C. J., Karlinsey, J. M., Bienvenue, J. M., Legendre, L. A., Roper, M. G., Feldman, S. H., Hughes, M. A., Hewlett, E. L., Merkel, T. J., Ferrance, J. P., Landers, J. P., "A fully integrated microfluidic genetic analysis system with sample-in-answer-out capability," Proc. Natl Acad. Sci. USA 2006, 103, 1927219277.

[6] Johnson, M. E., Landers, J. P., "Fundamentals and practice for ultrasensitive laserinduced fluorescence detection in microanalytical systems," Electrophoresis 2004, $25,3513-3527$.

[7] Schwarz, M. A., Hauser, P. C., "Recent developments in detection methods for microfabricated analytical devices," Lab Chip 2001, 1, 1-6.

[8] Kuswandi, B., Nuriman, Huskens, J., Verboom, W., "Optical sensing systems for microfluidic devices: a review," Anal. Chim. Acta 2007, 601, 141-155. 
[9] Psaltis, D., Quake, S. R., Yang, C., "Developing optofluidic technology through the fusion of microfluidics and optics. Nature 2006, 442, 381-386.

[10] Monat, C., Domachuk, P., Eggleton, B. J., "Integrated optofluidics: a new river of light," Nat. Photon. 2007, 1, 106-114.

[11] Verpoorte, E., “Chip vision - optics for microchips,” Lab Chip 2003, 3, 42-52.

[12] Yager, P., Edwards, T., Fu, E., Helton, K. Nelson K., Tam, M. R., Weigl, B. H., "Microfluidic diagnostic technologies for global public health," Nature 2006, 442, 381-393.

[13] Davis, K. M., Miura, K., Sugimoto, N., Hirao, K., "Writing waveguides in glass with a femtosecond laser," Opt. Lett. 1996, 21, 1729-1731.

[14] Gattass, R., Mazur, E., "Femtosecond laser micromachining in transparent materials," Nat. Photon. 2008, 2, 219-225.

[15] Martinez Vazquez, R., Osellame, R., Nolli, D., Dongre, C., van den Vlekkert, H. H., Ramponi, R., Pollnau, M., Cerullo, G., "Integration of femtosecond laser written optical waveguides in a lab-on-chip," Lab Chip 2009, 9, 91-96.

[16] Dongre, C., Dekker, R., Hoekstra, H. J. W. M., Pollnau, M., Martínez Vázquez, R., Osellame, R., Cerullo, G., Ramponi, R., van Weeghel, R., Besselink, G. A. J., van den Vlekkert, H. H., "Fluorescence monitoring of microchip capillary electrophoresis separation with monolithically integrated waveguides," Opt. Lett. $2008,33,2503-2505$.

[17] Bliss, C. L., McMullin, J. N., Backhouse, C. J., "Rapid fabrication of a microfluidic device with integrated optical waveguides for DNA fragment analysis," Lab Chip 2007, 7, 1280-1287. 
[18] Krawczyk, M. J., Dulak, J., Kulakowski, K., "Mean free path and peak dispersion in the geometration motion in gel electrophoresis," Electrophoresis 2002, 23, 182185.

[19] Boumans, P. W. J. M., "Detection limits and spectral interferences in atomic emission spectrometry," Anal. Chem. 1994, 66, 459-467.

[20] Paegel, B. M., Emrich, C. A., Wedemeyer, G. J., Scherer, J. R., Mathies, R. A., "High throughput DNA sequencing with a microfabricated 96-lane capillary array electrophoresis bioprocessor,” Proc. Natl Acad. Sci. 2002, 99, 574-579.

[21] Landers, J. P., "Molecular diagnostics on electrophoretic microchips," Anal. Chem. 2003, 75, 2919-2927.

[22] Dolnik, V., Gurske, W. A., "Capillary electrophoresis in sieving matrices: selectivity per base, mobility slope, and inflection slope," Electrophoresis 1999, 20, 3373-3380.

[23] Crespi, A., Gu, Y., Ngamsom, B., Hoekstra, H. J. W. M., Dongre, C., Pollnau, M., Ramponi, R., van den Vlekkert, H. H., Watts, P., Cerullo, G., Osellame, R., “Threedimensional Mach-Zehnder interferometer in a microfluidic chip for spatiallyresolved label-free detection," Lab Chip 2010, 10, 1167-1173. 


\section{Figure Captions}

Figure 1. Schematic of the optofluidic chip showing reservoirs 1-4, sample injection channel (reservoir $1 \rightarrow$ reservoir 2) and CE separation channel (reservoir $3 \rightarrow$ reservoir 4), as well as the integrated optical WG and detection window.

Figure 2. Movie recorded with a CCD camera showing transient fluorescence from several molecule plugs formed by CE separation of the DNA ladder as these plugs pass by the point of integrated-WG laser excitation at $488 \mathrm{~nm}$.

Figure 3. CE separation in an optofluidic chip of a DNA ladder consisting of doublestranded molecules with 17 different sizes (50-3000 base-pairs), fluorescence-labeled with an intercalating dye. (A) Electropherogram of fluorescence intensity vs. migration time recorded by a PMT after integrated-WG laser excitation. The a priori known basepair sizes are indicated; (B) repetition of the same experiment in the same microchip; (C) Gaussian fits to the fluorescence peaks of Fig. 3B, The peak widths are indicated; (D) peak widths obtained from Gaussian fits to the fluorescence peaks of Fig. 3A and 3B vs. square root of the base-pair size.

\footnotetext{
Figure 4. Fluorescence intensity vs. migration time for 12 double-stranded DNA molecules in the size range of $150-500 \mathrm{bp}$, flown and separated independently in a different microchip.
} 
Figure 5. For the two flow experiments on the same chip with 17 double-stranded DNA molecules in the size range of 50-3000 bp, and the flow experiment with the 12 doublestranded DNA molecules in the size range of 150-500 bp, in a different chip, the plots of (A) Migration time (on a linear scale) vs. DNA size (on a logarithmic scale), indicating a reproducibly high sizing accuracy; (B) Electrophoretic mobility vs. base-pair size; on a double logarithmic scale; (C) Migration time vs. base-pair size on a double logarithmic scale; depicting the different flow mechanisms. 


\section{Figure 1}

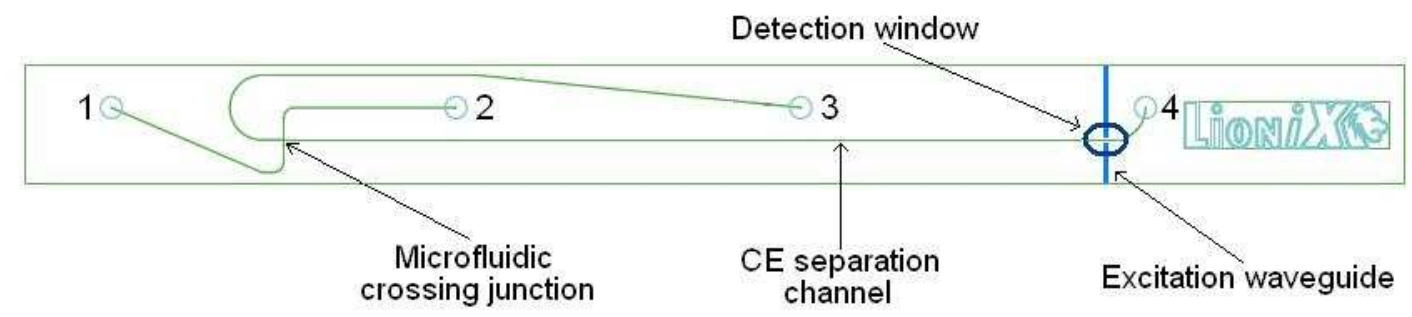

17

18

19

20

21

22

23

24

25

26

27

28

29

30

31

32

33

34

35

36

37

38

39

40

41

42

43

44

45

46

47

48

49

50

51

52

53

54

55

56

57

58

59

60

Wiley-ver 
Figure 2
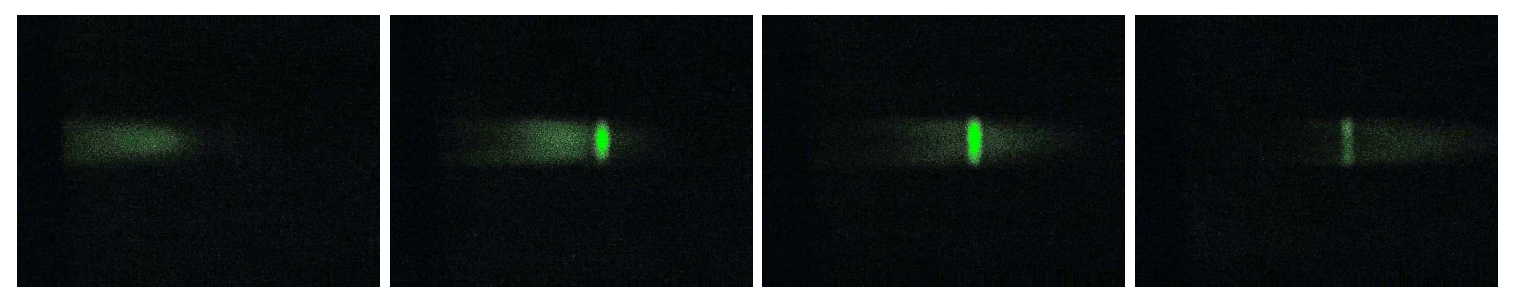

$$
\mathrm{t}=137 \mathrm{~s}
$$

$\mathrm{t}=137.5 \mathrm{~s}$

$\mathrm{t}=137.8 \mathrm{~s}$

$\mathrm{t}=138.2 \mathrm{~s}$ 
Figure 3

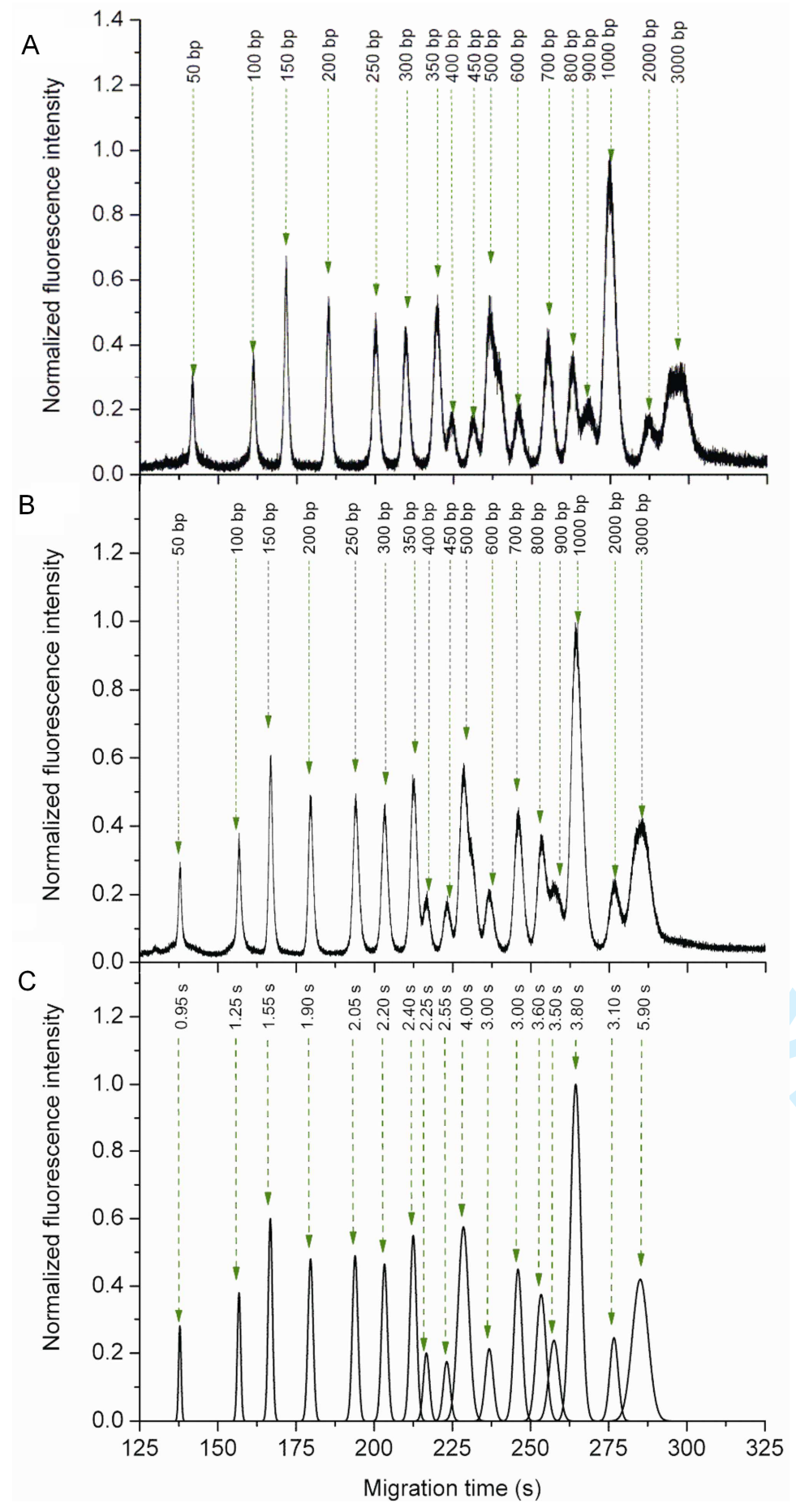

51

52

53

54

55

56

57

58

59

60 
D

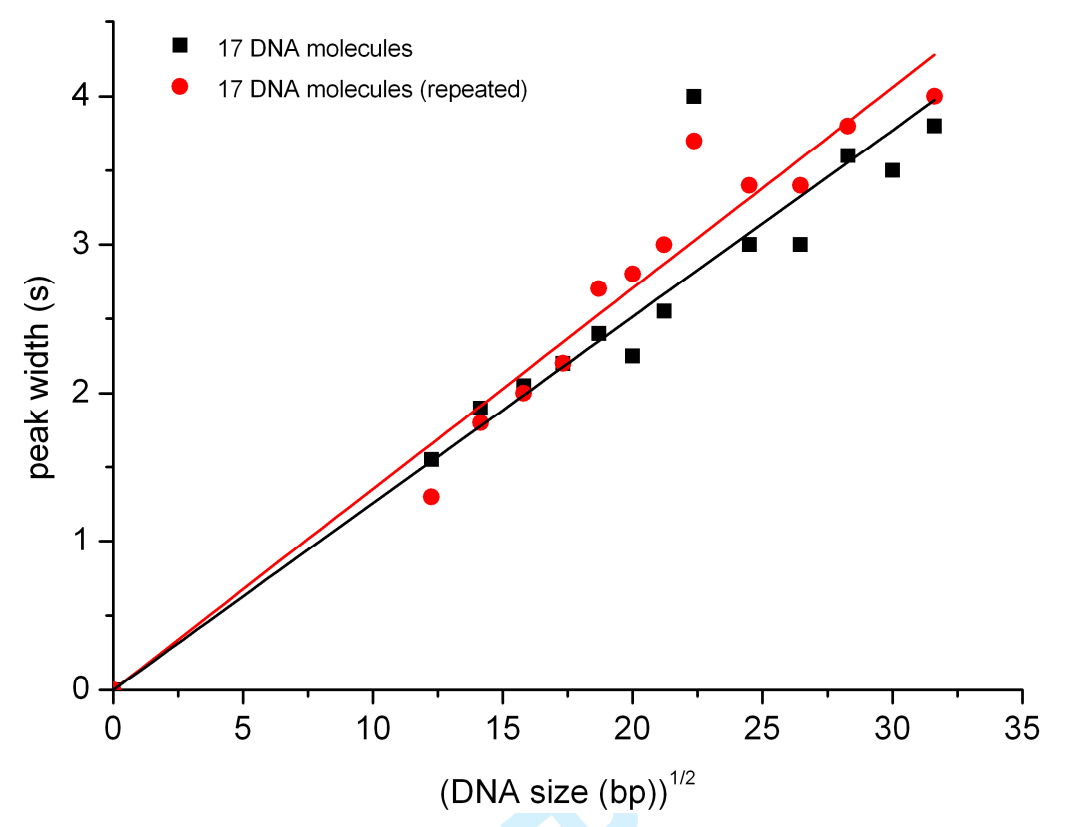

26

27

28

29

30

31

32

33

34

35

36

37

38

39

40

41

42

43

44

45

46

47

48

49

50

51

52

53

54

55

56

57

58

59

60

Wiley-vch 
Figure 4

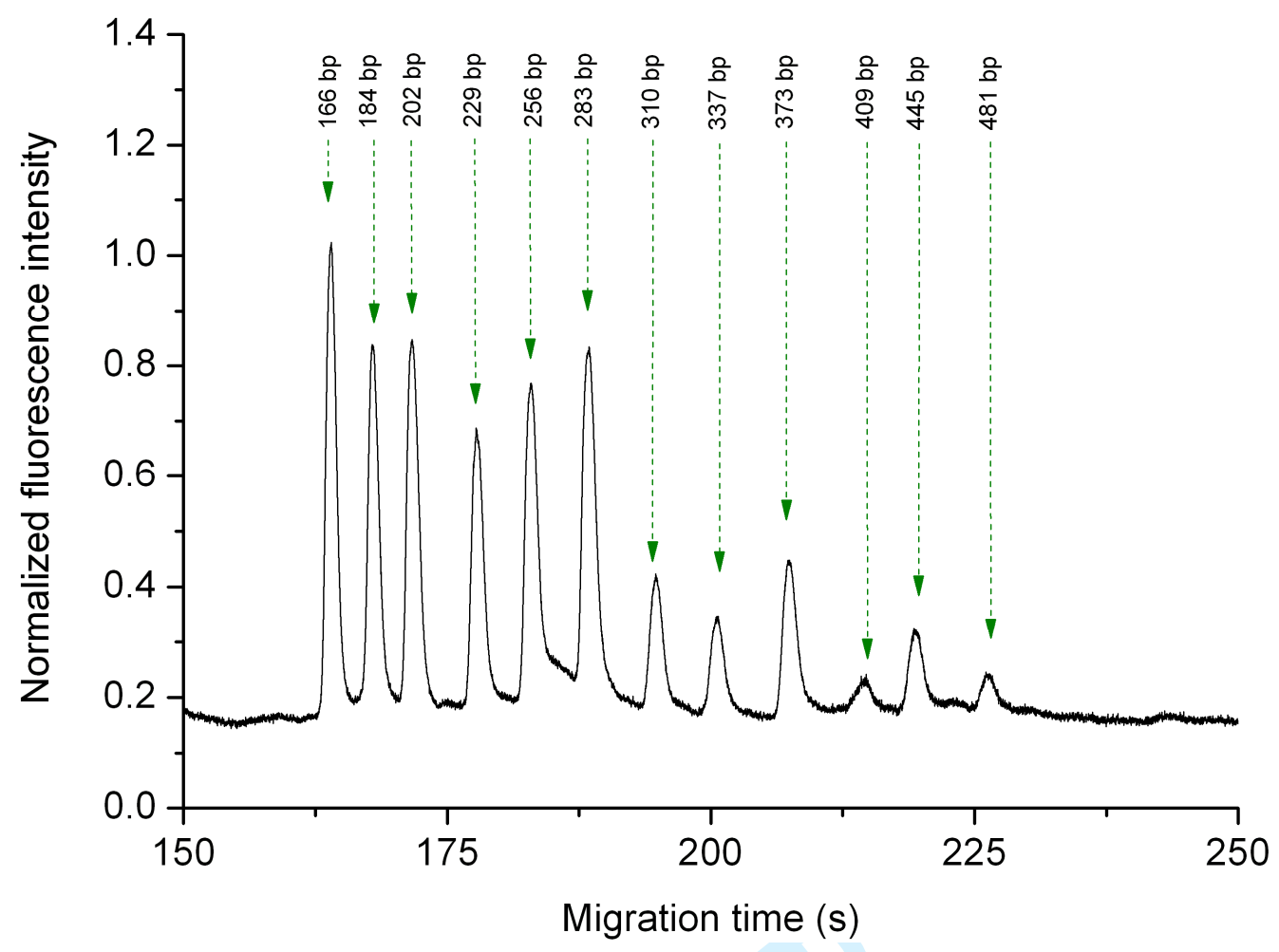

33

34

35

36

37

38

39

40

41

42

43

44

45

46

47

48

49

50

51

52

53

54

55

56

57

58

59

60 
Figure 5

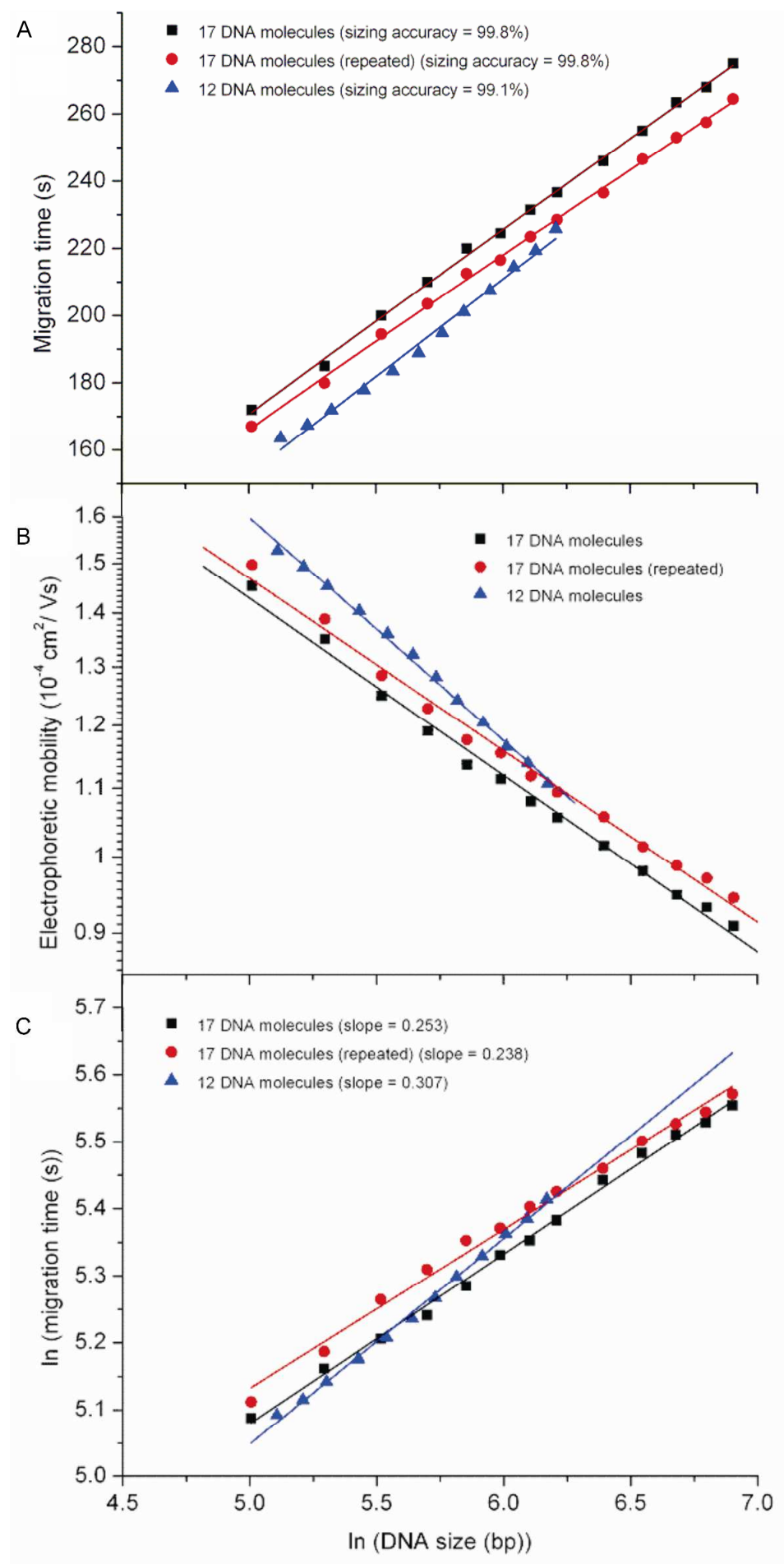

